\title{
Two medicolegal issues of concern
}

\section{Lundgren AC}

Head of Clinical Governance, Wits Donald Gordon Medical Centre; Honorary visiting Professor, Steve Biko Centre for Bioethics, University of the Witwatersrand

Corresponding author, email: chris.lundgren@wits.ac.za

\section{Unnatural Deaths: are we signing them off as "natural" deaths?}

\section{Health Professions Amendment Act No 29 of 2007':}

"Death of a person undergoing a procedure of therapeutic, diagnostic or palliative nature or of which any aspect of such procedure has been a contributory cause, shall not be deemed to be a death from natural causes as contemplated in the Inquests Act, 1959, or the Births, Marriages and Deaths Registration Act, 1963".

\section{Regulation 359 (March 2018) of the National Health Act 20032:}

These Regulations are called Regulations Regarding the Rendering of Forensic Pathology Service, 2018.

"Unnatural death" for the purposes of the medico-legal investigation of death, the following shall be deemed to be deaths due to unnatural causes, as contemplated in the Inquests Act 1959 (Act No. 58 of 1959):

(a) Any death due to physical or chemical influence, direct or indirect, or related complications;

(b) Any death, including those deaths which would normally be considered to be a death due to natural causes, which may have been the result of an act of commission or omission which may be criminal in nature;

(c) Any death as contemplated in section 56 of the Health Professions Act, 1974 (Act No. 56 of 1974) See amendment to the Act above; and

(d) Any death which is sudden and unexpected, or unexplained, or where the cause of death is not apparent.

\section{Unnatural Deaths in Health Establishments²:}

1. A person in charge of a health establishment, where a person has been declared dead and the cause of death appears to be due to unnatural causes must:

(a) Immediately notify the South African Police Service and the Service of such death;

(b) Preserve, provide access to and or make available of all the relevant medical paraphernalia, exhibits and applicable specimens, especially biological fluid specimens in the case of suspected toxicology cases; (c) Ensure access to and availability of all the deceased's full medical records including laboratory and investigative reports;

(d) Not hand over the body or items referred to in paragraphs (b) and (c) to an undertaker;

2. The medical records and or relevant completed clinical forms must accompany the deceased to the designated facility.

The patient using/abusing Anabolic Steroids.....is he/ she at risk for sudden death postoperatively?

An increasing number of young people are taking performing enhancing drugs, particularly regular gym attendees and recreational body-builders. The commonly used anabolic steroids comprise two main groups of drugs: the 17 a alkyl derivatives taken orally, and the parenteral $17 \beta$-ester derivatives. ${ }^{3}$

These agents may have the following effects, which may impact on anaesthesia ${ }^{3}$ :

1. Cardiovascular:

- Hypertension

- Left ventricular hypertrophy (resulting in diastolic dysfunction);

- Irreversible focal myocardial fibrosis (may act as a focus for malignant arrhythmias);

- Atheromatous coronary artery disease as a result of an abnormal lipid profile.

2. Blood components:

- Polycythemia

- Hypercoagulopathy (may result in cerebral thrombosis, left ventricular mural thrombus, thrombosis in peripheral arteries)

3. Hepatic:

- Liver dysfunction

- Hepatotoxicity

4. Large muscle mass and high caloric intake:

- May lead to increased oxygen consumption and carbon dioxide production. 
5. Neuropsychiatric effects, which may modify behaviour, as well as emergence from anaesthesia.

When assessing these patients preoperatively, it is important to check if they are also taking nutritional supplements and "accessory" drugs, such as ephedra, insulin, diuretics and growth hormone. ${ }^{3}$

Thus, despite a young body builder being assessed as an ASA 1 patient, if he/she is using or abusing anabolic steroids and other drugs, the anaesthetic may be fraught with problems.
1. No 29 of 2007: Health Professions Amendment Act 2007

2. No R359. No 61 of 2003: National Health Act

3. Kam PCA, Yarrow M. Anabolic steroid abuse. Anaesthesia. $2005 ; 60: 685-92$ 